01

\title{
Расчет спектра фоторекомбинации при облучении атомов сильным лазерным полем на основе марковского приближения
}

\author{
(C) A.М. Ишханян ${ }^{1,2}$, В.П. Крайнов ${ }^{3 \text { व }}$ \\ ${ }^{1}$ Российско-Армянский университет, \\ 0051 Ереван, Армения \\ ${ }^{2}$ Институт фризических исследований НАН Армении, \\ 0203 Аштарак, Армения \\ ${ }^{3}$ Московский фризико-технический институт (национальный исследовательский университет), \\ 141700 Долгопрудный, Россия \\ ฯ e-mail: vpkrainov@mail.ru
}

Поступила в редакцию 20.12.2021 г.

В окончательной редакции 20.12.2021 г.

Принята к публикации 30.12.2021 г.

В рамках марковского (факторизационного) приближения, основанного на факторизации полной матрицы плотности системы из атома, лазерного поля и электромагнитного поля вакуума, рассчитан спектр спонтанного излучения фотонов при облучении атомов сильным лазерным полем.

Ключевые слова: фемтосекундные лазерные импульсы, марковское приближение, туннельная ионизация, пондеромоторная энергия, спонтанное излучение.

DOI: $10.21883 /$ OS.2022.04.52257.46-21

\section{Введение}

В последние годы активно развивается трехступенчатая модель Коркума [1-3] для процесса облучении атомов сильными фемтосекундными лазерными импульсами. Первая ступень состоит в квантовой туннельной ионизации атома сильным линейно поляризованным лазерным излучением оптической частоты в условиях малого значения параметра Келдыша [4]. На второй ступени вылетевший электрон уходит далеко от образовавшегося материнского иона по сравнению с размерами иона. Примерно через полпериода лазерного поля возникает два варианта в зависимости от фазы электромагнитного поля в момент туннельной ионизации при вылете из-под туннельного барьера. В первом варианте электрон улетает на бесконечность, а его спектр кинетических энергий $E_{k}$ определяется в основном направлением движения электрона вдоль вектора поляризации лазерного поля и имеет вид $\exp \left(-2 E_{k} \gamma^{3} / 3 \hbar \omega\right)$. Здесь $\gamma=\omega \sqrt{2 m I_{p}} / e F \ll 1$ - параметр Келдыша, $I_{p}-$ потенциал ионизации атома. Далее $F, \omega-$ амплитуда и частота внешнего монохроматического электромагнитного поля соответственно, $m$ - масса электрона. Электрон поглощает индуцированно фотоны лазерного поля. Максимальная кинетическая энергия электрона при этом равна $2 E_{p}$. Здесь определена средняя колебательная (пондеромоторная) энергия электрона $E_{p}=(e F / 2 m \omega)^{2}$ Для сильного поля она велика по сравнению с потенциалом ионизации атома $I_{p}$. Малое отношение $I_{p} / E_{p} \ll 1$ представляет собой квадрат параметра Келдыша. Во втором варианте электрон поворачивает назад и возвращается к иону. При этом он также поглощает большое количество фотонов лазерного поля, и его энергия становится порядка средней колебательной (пондеромоторной) энергии электрона. Максимальная поглощенная вылетевшим электроном энергия равна $3.173 E_{p}$ [1]. На третьей ступени возникает два возможных варианта. В первом варианте электрон упруго рассеивается на материнском ионе и улетает на бесконечность. При этом его максимальная кинетическая энергия после поглощения дополнительных фотонов внешнего электромагнитного поля равна приблизительно $10 E_{p}$. Во втором варианте энергия электрона тратится на испускание спонтанного фотона высокой энергии в процессе фоторекомбинации электрона в основное состояние атома $n$. Переход в возбужденные состояния атома гораздо менее вероятен.

Процесс туннельной ионизации может быть описан также как поглощение большого количества фотонов. В настоящей работе рассматривается именно второй вариант третьей ступени модели Коркума. В соответствии с дипольными правилами отбора поглощается нечетное количество фотонов, и энергия спонтанного фотона равна $N \hbar \omega, N \gg 1$. Возможно и поглощение четного количества фотонов, когда конечное состояние атома находится в непрерывном спектре [5] - это процесс неупругого рассеяния электрона на третьем этапе. Процессы спонтанного излучения фотонов в сильном электромагнитном поле рассматривались в работах [6-9]. Для вероятности спонтанной рекомбинации в единицу времени применима формула Штоббе [10], получаемая квазиклассическим обобщением известной 
формулы Штоббе для атома водорода:

$$
\gamma=\frac{128 \pi}{3} \frac{\hbar^{2}}{m e^{2}}\left(\frac{e^{2}}{\hbar c}\right)^{3}\left(\frac{I_{p}}{N \hbar \omega}\right)^{5 / 2} n \sqrt{\frac{2 N \hbar \omega}{m}}
$$

Здесь $n-$ концентрация электронов. Задача настоящей работы состоит в определении энергетического спектра испущенных спонтанных фотонов в такой двухуровневой модели, состоящей из основного состояния атома $n$ с практически нулевой энергией и высоковозбужденного состояния непрерывного спектра $m$ с энергией $N \hbar \omega$, $N \gg 1$.

\section{Теоретическая часть}

В случае однофотонного процесса испускания спонтанного фотона в двухуровневой системе такая задача была решена в работе [11]. Полная матрица плотности системы из атома, лазерного поля и электромагнитного поля вакуума представлялась в виде произведения матрицы плотности атома в поле и матрицы плотности для операторов фотонов электромагнитного поля. Это упрощение задачи называется марковским (или факторизационным) приближением. Оно применимо в случае $\Gamma \tau \gg 1$, где $\Gamma-$ спонтанная ширина, а $\tau$ - продолжительность лазерного фемтосекундного импульса. Физика указанного приближения состоит в том, что изменения, происходящие с атомом при его взаимодействии с лазерным импульсом, не влияют на вакуумные состояния электромагнитного поля. В работе [12] мы рассматривали противоположный предельный случай $Г \tau \ll 1$, который не требует введения матрицы плотности.

Процесс спонтанного испускания фотона может быть записан в терминах упругого и неупругого рассеяния фотонов лазерного поля на атоме. Эти два процесса принципиально отличаются друг от друга. Упругое (несмещенное) рассеяние в слабом внешнем поле это основной процесс. Он является фактически классическим и представляет собой резонансную флуоресценцию. Частота испущенного фотона в слабом поле строго равна частоте падающего фотона, близкой к частоте атомного перехода в двухуровневой системе. Соответственно спектр представляет собой один профиль Лоренца со спонтанной шириной. Упругое рассеяние определяется квадратом среднего дипольного момента атома. В сильном лазерном поле спектр несмещенного рассеяния представляет собой два максимума с расщеплением между ними, равным частоте Раби [13]. Это имеет место в сильном поле, когда частота Раби больше спонтанной ширины. Соответствующая диаграмма Фейнмана содержит поглощение лазерного фотона с возбуждением атома, затем его испускание с возвращением атома в исходное состояние, затем снова поглощение лазерного фотона и, наконец, испускание спонтанного фотона, частота которого строго равна частоте $\omega$.

Однако в сильном поле гораздо больший вклад вносит не квадрат среднего значения дипольного момента, а среднее значение квадрата дипольного момента, т.е. его дисперсия. Это определяет так называемое смещенное рассеяние. Оно является чисто квантовым процессом. Для смещенного рассеяния диаграмма Фейнмана состоит в поглощении лазерного фотона $\omega$, испускании спонтанного фотона $v_{1}$, снова в поглощении лазерного фотона $\omega$ и затем в испускании второго спонтанного фотона $v_{2}$. При этом, согласно закону сохранения энергии, $v_{1}+v_{2}=2 \omega$ и частота спонтанного фотона может отличаться от $\omega$ в отличие от несмещенного рассеяния.

В сильном поле надо суммировать все диаграммы Фейнмана и более высокого порядка, например, диаграмму, в которой к предыдущей диаграмме надо добавить еще поглощение лазерного фотона и испускание спонтанного фотона. Закон сохранения энергии в этом случае имеет вид $v_{1}+v_{2}+v_{3}=3 \omega$. Таких диаграмм будет бесконечное количество. Их суммирование достигается в марковском приближении решением уравнения для двухвременной матрицы плотности. Это и сделано в работе [11] в случае однофотонного перехода. Операторы рождения и поглощения вакуумных фотонов берутся в представлении Гейзенберга. При вычислении флуктуации дипольного момента возникают элементы матрицы плотности в различные времена

$$
\begin{aligned}
\left\langle\left|\hat{\mathbf{d}}_{V}\right|^{2}\right\rangle= & \left\langle\int_{-\infty}^{\infty}\left|\mathbf{d}_{m n}\right|^{2}\left\langle\hat{\rho}_{n m}(t) \hat{\rho}_{m n}(t+\tau)\right\rangle\right. \\
& \times \exp (-i \nu \tau) d \tau+c . c .\rangle .
\end{aligned}
$$

Здесь усреднение проводится по времени $t$.

Однако операторы в представлении Гейзенберга обладают стандартными коммутационными свойствами только в одинаковые моменты времени. Поэтому элементы матрицы плотности в сдвинутый момент времени надо разлагать в ряд по полной системе этих элементов в несдвинутый момент времени. Для коэффициентов разложения получается система линейных уравнений. Она имеет тот же вид, что и исходная система уравнений для элементов матрицы плотности, но с другими начальным условиями:

$$
\begin{gathered}
i \dot{\rho}_{n n}=i \gamma \rho_{m m}+V(t)\left(\rho_{m n}-\rho_{n m}\right), \\
i \dot{\rho}_{n m}=-(i / 2) \gamma \rho_{n m}+\omega_{n m} \rho_{n m}+V(t)\left(\rho_{m m}-\rho_{n n}\right), \\
i \dot{\rho}_{m n}=-(i / 2) \gamma \rho_{m n}+\omega_{m n} \rho_{m n}+V(t)\left(\rho_{n n}-\rho_{m m}\right), \\
\rho_{m m}+\rho_{n n}=1 .
\end{gathered}
$$

Решение такой системы в общем случае громоздко из-за формул Кардано для корней характеристических кубических уравнений. Упрощение достигается в очень сильном поле, когда частота Раби велика по сравнению со спонтанной шириной, и при нулевой расстройке резонанса в двухуровневой системе.

В рассматриваемой нами задаче нулевая расстройка резонанса достигается естественным путем, так как 


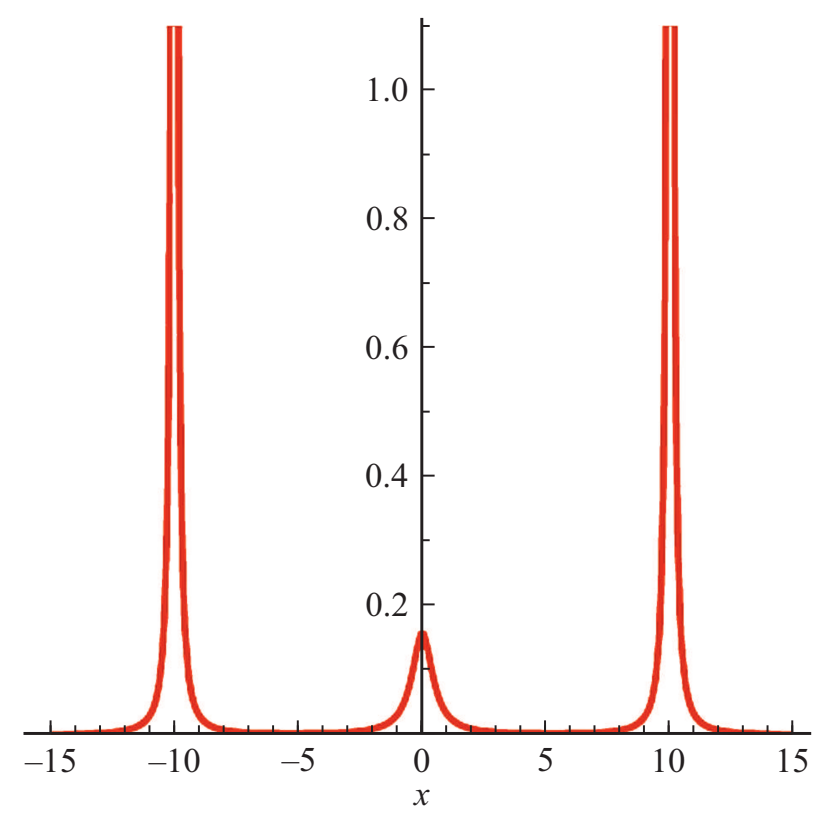

Спектр испущенных спонтанных фотонов.

энергия состояния непрерывного спектра $m$ на третьей ступени модели Коркума отличается от энергии начального связанного состояния $n$ точно на $N \hbar \omega$. Что касается частоты Раби, то ее надо заменить на многофотонный матричный элемент [13], который мы обозначим как $V$, не конкретизируя его явное выражение.

Для частотного спектра испущенных спонтанных фотонов, решая систему (3), получаем из (2) выражение, аналогичное тому, что было получено в [11] в однофотонном случае:

$$
\begin{aligned}
& d w(v)=\frac{\gamma^{2} d v}{2 \pi}\left\{\frac{3}{16(v-N \omega+V)^{2}+9 \gamma^{2}}\right. \\
& \left.+\frac{1}{4(v-N \omega)^{2}+\gamma^{2}}+\frac{3}{16(v-N \omega-V)^{2}+9 \gamma^{2}}\right\} .
\end{aligned}
$$

Величина $\gamma$ определяется формулой (1). Спектр, рассчитанный для $V / \gamma=10$, показан на рисунке. По оси абсцисс отложена величина $x=(v-N \omega) / \gamma$. Из рисунка видно, что интенсивности сателлитов Раби значительно больше, чем центральный максимум. При интегрировании (3) по частотам фотонов получим $w=\gamma / 2$. Это соответствует испусканию спонтанного фотона из состояния непрерывного спектра, заселенного при нулевой расстройке резонанса с вероятностью $1 / 2$.

\section{Заключение}

В настоящей работе мы исследовали возможность наблюдения триплета Моллоу при спонтанном излучении в процессе облучения атомов сильными фемтосекундными лазерным импульсами. Для наблюдения этого эффекта ширина интервала частот лазерного импульса должна быть достаточно мала по сравнению с многофотонной частотой Раби. В работе [14] мы исследовали аналитически выход спонтанных фотонов при туннельной ионизации атомов интенсивным низкочастотным лазерным излучением вблизи классического порога $N_{\max } \hbar \omega=3.173 E_{p}$ на основе трехступенчатой модели Коркума. Была получена колоколообразная форма универсального фотонного энергетического спектра с максимумом при частоте спонтанного фотона $0.65 N_{\max } \omega$. Поэтому в возможном эксперименте энергию непрерывного спектра следует подбирать в окрестности этого значения.

\section{Финансирование работы}

Исследование выполнено за счет грантов Российского фонда фундаментальных исследований (проект № 20-52-05012), Комитета по науке Армении (грант № 20RF-171) и Армянского национального фонда науки и образования (грант № PS-5701).

\section{Конфликт интересов}

Авторы заявляют, что у них нет конфликта интересов.

\section{Список литературы}

[1] P.B. Corkum. Phys. Rev. Lett., 71 (13), 1994 (1993).

[2] F. Krausz, M. Ivanov. Rev. Mod. Phys., 81 (1), 163 (2009).

[3] G. Orlando, T.-S. Ho, Sh.-I. Chu. J. Opt. Soc. Am., 37 (5), 1500 (2020).

[4] Л.В. Келдыш. ЖЭТФ, 47 (5), 1945 (1964).

[5] A.V. Bogatskaya, E.A. Volkova, A.M. Popov. Las. Phys. Lett., 14 (5), 055301 (2017).

[6] Р.В. Карапетян, М.В. Федоров. ЖЭТФ, 73 (3), 516 (1978).

[7] В.Д. Кондратович, В.Н. Островский. ЖЭТФ, $79(2), 395$ (1980).

[8] D.B. Milosevic, F. Ehlotzky. J. Phys. B, 36, 373 (2003).

[9] A.V. Flegel, M.V. Frolov, N.L. Manakov, A.N. Zheltukhin. J. Phys. B, 42, 241002 (2009).

[10] M. Stobbe. Annalen der Physik, 399 (6), 661 (1930).

[11] B.R. Mollow. Phys. Rev., 188 (5), 1969 (1969).

[12] D.N. Yangaliev, V.P. Krainov, O.I. Tolstikhin. Phys. Rev. A, 101 (1), 013410 (2020).

[13] N.B. Delone, V.P. Krainov. Multiphoton Processes in Atoms, 2nd ed. (Springer, Berlin, 2000), ch. 6.

[14] A.V. Gets, V.P. Krainov. Las. Phys. Lett., 15(1), 016005 (2018). 